Mots. Les langages du politique

$87 \mid 2008$

Chrononymes. La politisation du temps

\title{
Onomastique politique. Éléments de bibliographie
}

Paul Bacot, Laurent Douzou et Jean-Paul Honoré

\section{OpenEdition}

\section{Journals}

Édition électronique

URL : https://journals.openedition.org/mots/12552

DOI : 10.4000/mots.12552

ISSN : 1960-6001

\section{Éditeur}

ENS Éditions

\section{Édition imprimée}

Date de publication : 21 juillet 2008

Pagination : 85-95

ISBN : 978-2-271-06685-5

ISSN : 0243-6450

\section{Référence électronique}

Paul Bacot, Laurent Douzou et Jean-Paul Honoré, « Onomastique politique. Éléments de

bibliographie », Mots. Les langages du politique [En ligne], 87 | 2008, mis en ligne le 21 juillet 2010,

consulté le 24 avril 2022. URL : http://journals.openedition.org/mots/12552 ; DOI : https://doi.org/

$10.4000 /$ mots. 12552 


\section{Paul Bacot, Laurent Douzou, Jean-Paul Honoré}

\section{Onomastique politique. Éléments de bibliographie}

Cette bibliographie (francophone) comporte des références à des travauxutiles à l'étude de la dimension politique des noms propres (chrononymes, toponymes, anthroponymes, praxonymes et ergonymes). À quelques exceptions près, elle ne mentionne pas les travaux de portée générale consacrés auxnoms propres, malgré leur évidente utilité en la matière. Elle privilégie les études portant sur des objets de l'histoire moderne et, surtout, contemporaine.

AKIN Salih, 2000, "Ne dites pas Kurde, dites citoyen turc», Mots. Les langages du politique, $\mathrm{n}^{\circ} 64$, décembre, Autour d'une crise franco-australienne. Stéréotypies xénophiles et xénophobes, p. 130-135.

AKın Salih éd., 1999, Noms et re-noms. La dénomination des personnes, des langues et des territoires, Rouen, Publications de l'Université de Rouen.

Amalvı Christian, 1997, "Le 14 juillet. Du Dies irae à Jour de fête», Pierre Nora éd., Les lieux de mémoire, t. 1, La République, Paris, Gallimard, p. 383-420.

Atou Brahim, Benramdane Farid éd., 2005, Toponymie et anthroponymie de l'Algérie. Recueil bibliographique général, Oran, Crasc.

BACOT Paul, 1994, Dictionnaire du vote. Élections et délibérations, Lyon, Presses universitaires de Lyon.

BACOt Paul, RÉMI-GIRAUd Sylvianne éd., 2007, Mots de l'espace et conflictualité sociale, Paris, L'Harmattan (Langue et parole).

BACOT Paul, Douzou Laurent, Honoré Jean-Paul éd., 2008, Mots. Les langages du politique, $\mathrm{n}^{\circ} 87$, juillet, Chrononymes. La politisation du temps.

BACOT Paul, Douzou Laurent, HonorÉ Jean-Paul, 2008, "Onomastique politique. Éléments de bibliographie», Mots. Les langages du politique, $\mathrm{n}^{\circ} 87$, juillet, Chrononymes. La politisation du temps, p. 85-95.

BARBÉRIS Jeanne-Marie, 2007, "Quartier des uns, quartier des autres. La construction de l'espace urbain dans la parole», P. Bacot, S. Rémi-Giraud éd., Mots de l'espace et conflictualité sociale, Paris, L'Harmattan, p. 187-197.

BASSEt Louis, 2007, "Des “Peuples de la Mer" à l'Atlantide», P. Bacot, S. RémiGiraud éd., Mots de l'espace et conflictualité sociale, Paris, L'Harmattan.

BAUER Laurent, 2001, «De la diachronie à la synchronie. Étude de la dénomination des voies à Cergy-Pontoise », Langage et société, $n^{\circ}$ 96, juin, p. 9-27.

Université de Lyon, Institut d'études politiques, CNRS (Triangle), Paul.Bacot@univ-lyonz.fr Université de Lyon, Institut d'études politiques, CNRS (LAHRA), laurent.douzou@univ-lyon2.fr Université Paris 12 Val-de-Marne, CEDITEC, jp.honore@wanadoo.fr 
Baylon Christian, Fabre Paul, 1982, Les noms de lieux et de personnes, Paris, Nathan.

Bedoucha-Albergonı Geneviève, 1980, "La mémoire et l'oubli. L'enjeu du nom dans une société oasienne», Annales. Histoire, Sciences sociales, vol. 35, $\mathrm{n}^{\circ}$ 3-4, p. 730-747.

Bellescize Diane, 1993, "De l'Acte additionnel aux Constitutions de l'Empire, 22 avril 1815. Une Constitution mal nommée pour un régime sans nom », Revue de droit public et de science politique, $\mathrm{n}^{\circ}{ }_{4}$, août, p. 1043-1077.

Bellevaire Patrick, 2001, "Les noms d'Okinawa. Une japonité singulière», Mots. Les langages du politique, $n^{\circ} 66$, juillet, Discours d'Asie. Identités et ruptures, p. 71-88.

Bernardino Palumbo, 1992, "Le même sang, le même nom, la même terre. Théories traditionnelles de l'identité sociale dans un village du Sannio », Mélanges de l'École française de Rome. Italie et Méditerranée, ${ }^{\circ} 104$ (2), p. 643-693.

BERNET Jacques, 1980, "Les prénoms républicains sous la Révolution française. L'exemple du district de Compiègne, 1793-1795», A. Bideau, M.-E. Ducreux, J. Dupâquier éd., Le prénom. Mode et histoire, Paris, EHESS, p. 247-253.

BERTRAND Régis, 2001, "Aux grands hommes, la mairie reconnaissante”. Hommes illustres et noms de rues à Marseille vers 1770-1870», J.-C. Bouvier, J.-M. Guillon éd., La toponymie urbaine. Significations et enjeux, Paris, L'Harmattan, p. 65-73.

BIANCHI Serge, 1984, "Les prénoms “révolutionnaires" en l'an II. L'exemple de Corbeil et de la Seine-et-Marne», A. Bideau, M.-E. Ducreux, J. Dupâquier éd., Le prénom. Mode et histoire, Paris, EHESS, p. 255-270.

BIDEAU Alain, Ducreux Marie-Élisabeth, DUPÂQUIER Jacques éd., 1984, Le prénom. Mode et histoire. Les Entretiens de Mahler 1980, Paris, EHESS.

Billy Pierre-Henri, 1994, "Typologie du surnom personnel», Nouvelle revue d'onomastique, $\mathrm{n}^{\circ} 23-24$, p. 13-30.

Bizet Ange, 2003, «Timor, l'orientale», Mots. Les langages du politique, $n^{\circ} 71$, mars, Mondialisation(S), p. 157-166.

BOCQUET Denis, 2001, «Dénomination, négociation et spéculation. Le quartier des Prati di Castello à Rome, 1870-1911, J.-C. Bouvier, J.-M. Guillon éd., 2001, La toponymie urbaine. Significations et enjeux, Paris, L'Harmattan, p. 189-195.

BödEKER Hans-Erich, HinRICHS Ernst éd., 1991, Alteuropa - Ancien Régime - Frühe Neuzeit. Probleme und Methoden der Forschung, Stuttgart-Bad-Cannstatt.

BoIDIN Capucine, 2007, « Du Gran Líder Stoessner (1954-1989) au Karai Tendota Nicanor Duarte (2003-2006)», Mots. Les langages du politique, n 85 , novembre, Violence et démocratie en Amérique latine, p. 11-22.

Bonnafous Simone, Dahlem Jacqueline éd., 1997, Mots. Les langages du politique, $n^{\circ}{ }_{53}$, décembre, La Nouvelle-Calédonie après les accords de Matignon.

Bonnafous Simone, Honoré Jean-Paul, Tournier Maurice, 1985, "La désignation politique en France de 1879 à 1914 », A. Gérald, M. Robert éd., Histoire de la langue française, 1880-1914, Paris, CNRS, p. 41-98.

BosA Bastien, 2005, «Les mots et les choses. Les Aborigènes et la décolonisation », Genèses, n 61, décembre, p. 98-120. 
BouviER Jean-Claude, GUILLon Jean-Marie, 2001, La toponymie urbaine. Significations et enjeux, Paris, L'Harmattan.

BOYER Henri, 2008, "Fonctionnements sociolinguistiques de la dénomination toponymique», Mots. Les langages du politique, $\mathrm{n}^{\circ}$ 86, février, Toponymes. Instruments et enjeux, p. 9-21.

Boyer Henri, Paveau Marie-Anne éd., 2008, Mots. Les langages du politique, $\mathrm{n}^{\circ} 86$, février, Toponymes. Instruments et enjeux.

Bozon Michel, 1987, "Histoire et sociologie d'un bien symbolique, le prénom», Population, $\mathrm{n}^{\circ} 42$ (1), p. 83-98.

Branca-Rosoff Sonia, LeImdorfer François éd., 2001, Langage et société, n 96 , septembre, Espaces urbains. Analyses lexicales et discursives.

BRAnCA-Rosoff Sonia, Wionnet Chantal, 1995, «Le mot Dieu à travers les dictionnaires, du Trévoux au Petit Robert», Lexique, n 12-13, Actes du colloque Dictionnaires et littératures (1830-1990), 26-28 septembre 1999, Université de Lille 3, p. 229-239.

BRoccolıNI Alessandra, 2001, "Toponymie et identité à Naples. La napolanité du quartier Santa-Lucia, xıxe-xxe siècle », J.-C. Bouvier, J.-M. Guillon éd., La toponymie urbaine. Significations et enjeux, Paris, L'Harmattan, p. 171-187.

Bromberger Christian, 1982, "Pour une analyse anthropologique des noms de personnes», Langages, $n^{\circ}$ 66, p. 103-124.

BücHI Eva, 1996, Les structures du Französisches Etymologisches Wörterbuch, Tübingen, Niemeyer.

CALABRESE Steimberg Laura, 2006, «La construction de la mémoire historico-médiatique à travers les désignations d'événements », Travaux du Cercle belge de linguistique, $\mathrm{n}^{\circ}$ 1, p. 1-16. http://webho1.ua.ac.be/linguist/online/paps2006/cal2006.pdf

CALVET Louis-Jean, 1994, Les voies de la ville. Introduction à la sociolinguistique urbaine, Paris, Payot.

CAmbon Emmanuelle, 2000, “Jean-Marie Le Pen” / “Mégret”. La scission vue par le Front national », Mots. Les langages du politique, $n^{\circ}$ 63, juillet, Noms propres, p. 134-140.

- 2004, "Personnification et personnalisation dans le discours politique du Front national. Approche discursive de la figure du représentant politique construite par les formes d'anthroponymes », thèse de doctorat, Université de Paris 3.

CANUt Cécile, 2002, "Pouvoir, places et filiation. Les Senankuya au Mali », Cahiers de praxématique, $\mathrm{n}^{\circ} 38$, p. 175-197.

Cassanas Armelle, Demange Aude, Laurent Bénédicte, Lecler Aude éd., 2004, Dialogisme et nomination, Montpellier, Presses universitaires de Montpellier 3.

Cassanas Armelle, Demange Aude, Dutilleul-Guerroudj Élise, Laurent Bénédicte, LECLER Aude, 2004, "Repérage en diachronie des effets des stratégies idéologiques sur les objets du discours socio-politique», A. Cassanas, A. Demange, B. Laurent, A. Lecler éd., Dialogisme et nomination, Montpellier, Presses universitaires de Montpellier 3, p. 65-97.

Cavalla Cristelle, 2007, «Nord et Sud. De nouvelles définitions par les écologistes Les Verts?», P. Bacot, S. Rémi-Giraud, Mots de l'espace et conflictualité sociale, Paris, L’Harmattan, p. 233-241. 
Cheriguen Foudil, 1987, "Barbaros ou Amazigh. Ethnonymes et histoire politique en Afrique du Nord», Mots. Les langages du politique, $\mathrm{n}^{\circ}{ }_{15}$, octobre, Comment nommer?, p. 7-22.

- 1994, «Anthropo-toponymie et désignation de l'“environnement politique” », Mots. Les langages du politique, $\mathrm{n}^{\circ}$ 39, juin, Environnement, écologie, Verts, p. 93-98.

- 1998, «Nommer pour exister. De l'ethnonyme comme enjeu politique», Mots. Les langages du politique, $\mathrm{n}^{\circ} 57$, décembre, Algérie en crise. Entre violence et identité, p. 29-37.

ChRISTIN Anne-Marie éd., 1998, L'écriture du nom propre, Paris, L'Harmattan.

CHRISTIN Olivier, 2008, "Ancien Régime. Pour une approche comparatiste du vocabulaire historiographique», Mots. Les langages du politique, $n^{\circ} 87$, juillet, Chrononymes. La politisation du temps, p. 13-25.

Cislaru Georgeta, 2003, "La dénomination entre langue et discours. Sémantique et discursivité de la désignation du pays dans le discours de presse », Communication aux Rencontres de l'école doctorale Langage et Langues (ED 268), mai 2003, Université Paris 3 Sorbonne nouvelle, disponible sur le site des RJC, www.cavi. univparis3.fr/lpga/ED/activites/RJC2003_actes/index.html.

- 2004, "Le nom de pays dialogique dans la construction de l'événement médiatique», A. Cassanas, A. Demange, B. Laurent, A. Lecler éd., Dialogisme et nomination, Montpellier, Presses universitaires de Montpellier 3, p. 113-127.

- 2005, "Étude sémantique et discursive du nom de pays dans la presse française», thèse de doctorat en sciences du langage, Université de la Sorbonne nouvelle Paris 3.

- 2006, "Nom de pays et autoreprésentation dans le discours des périodiques nationaux français, anglophones, roumanophones et russes», Les Carnets du Cediscor, ${ }^{\circ}$ 9, p. 131-144.

- 2006, «Nom de pays, nom de peuple. Quels usages, quelles identités?», Cahiers de sociolinguistique, $\mathrm{n}^{\circ}$ 11, p. 131-144.

- 2007, "Reconstruction discursive d'un espace géopolitique. La Moldavie après 1991 », P. Bacot, S. Rémi-Giraud éd., Mots de l'espace et conflictualité sociale, Paris, L'Harmattan, p. 223-232.

- 2008, "Le nom de pays comme outil de représentation sociale», Mots. Les langages du politique, $\mathrm{n}^{\circ} 86$, Toponymes. Instruments et enjeux, p. 53-64.

Cislaru Georgeta, GuÉrin Olivia, Katia Morim, Née Émilie, Pagnier Thierry, Veniard Marie éd., 2007, L'acte de nommer. Une dynamique entre langue et discours, Paris, Presses de la Sorbonne nouvelle.

Corcuff Stéphane, 2005, «Taïwan existe-t-elle? 3, Du débat constitutionnel. Nom de régime et territoire national ", Monde chinois, ${ }^{\circ}{ }_{5}$, été-automne, p. 7-17.

Cortier Claude, 2007, «L'opposition Nord-Sud dans un corpus plurisémiotique latinoaméricain. Contribution à la compréhension des territoires idéologiques et culturels et des antagonismes géopolitiques », P. Bacot, S. Rémi-Giraud éd., Mots de l'espace et conflictualité sociale, Paris, L'Harmattan, p. 243-256. 
Cyr Danielle, Nagugwes Metallic Emmanuel, 1999, «Fantômes choronymiques de la dépossession", S. Aki éd., Noms et re-noms. La dénomination des personnes, des langues et des territoires, Rouen, Publications de l'Université de Rouen, p. 151-163.

DAHLEM Jacqueline, 1997, "Des historiens à la recherche d'un consensus », Mots. Les langages du politique, $\mathrm{n}^{\circ} 53$, décembre, La Nouvelle-Calédonie après les accords de Matignon, p. 26-47.

DAROt Mireille, 1997, "Calédonie, Kanaky ou Caillou? Implicites identitaires dans la désignation de la Nouvelle-Calédonie», Mots. Les langages du politique, $\mathrm{n}^{\circ} 53$, décembre, La Nouvelle-Calédonie après les accords de Matignon, p. 8-25.

DÉCIMO Marc, FIALA Pierre, 2004, "Michel Bréal, le marathon, l'olympisme et la paix», Mots. Les langages du politique, $n^{\circ} 76$, novembre, Guerres et paix. Débats, combats, polémiques, p. 127-135.

Delattre Éric, 2007, "Le changement de nom des communes françaises. Aspects économiques, marketing et stratégiques ", Revue d'économie régionale et urbaine, $\mathrm{n}^{\circ} 2$, p. $269-291$.

Deluz Ariane, 1967, «Anthroponymie et recherche historique», L'Homme, $n^{\circ} 7$ (1), p. 32-49.

DeRrIDA Jacques, 1984, Otobiographies. L'enseignement de Nietzsche et la politique du nom propre, Paris, Galilée.

DUCLERT Vincent, PROCHASSON Christophe éd., 2007 [2002], Dictionnaire critique de la République, Paris, Flammarion.

Dufour Françoise, Dutilleul-guerroud Élise, Laurent Bénédicte, 2005, La nomination. Quelles problématiques, quelles orientations, quelles applications?, Montpellier, Presses universitaires de la Méditerranée (Langue et praxis).

DURAND Guillaume, 2002, "Survivance des patronymes d'origine africaine à la Martinique chez les esclaves et les affranchis avant et après l'abolition de 1848 ", Nouvelle revue d'onomastique, $\mathrm{n}^{\circ}$ 39-40, p. 247-305.

Faron Olivier, PIllepich Alain, 1993, "Rue, îlot, quartier. Sur l'identification des espaces citadins à Milan au début du xıxe siècle », Mélanges de l'École française de Rome. Italie et Méditerranée, $\mathrm{n}^{\circ} 105$ (2), p. 333-348.

FAure Guy, 2001, "Les mots d'Asie. Essai de terminologie géopolitique», Mots. Les langages du politique, $\mathrm{n}^{\circ} 66$, juillet, Discours d'Asie. Identités et ruptures, p. 8-18.

FIALA Pierre, 1996, «Ex-Yougoslavie. Les noms aussi sont morcelés», Mots. Les langages du politique, $\mathrm{n}^{\circ}$ 47, juin, Les médias dans le conflityougoslave, p. 144-147.

- 2002, "Motivés, motivé», Mots. Les langages du politique, $\mathrm{n}^{\circ} 70$, novembre, La politique en chansons, p. 133-137.

Fiala Pierre, Rennes Juliette, 2002, "Majorité plurielle. Trajectoire d'une formule», Mots. Les langages du politique, $n^{\circ} 68$, mars, Les métamorphoses spatiales en politique, p. 123-130.

FICHARD François, 2005, «Les dénominations partisanes. Imposition d’un sens légitime par un discours politique catégorisant», F. Dufour, É. Dutilleul-Guerroudj, B. Laurent éd., La nomination. Quelles problématiques, quelles orientations, quelles applications?, Montpellier, Presses universitaires de la Méditerranée (Langue et praxis). 
FRAGNON Julien, 2007, "Quand le 11-Septembre s'approprie le onze septembre. Entre dérive métonymique et antonomase», Mots. Les langages du politique, $n^{\circ} 85$, novembre, Violence et démocratie en Amérique latine, p. 83-95.

Fragnon Julien, LAmy Aurélia, 2008, "L'Après-11 septembre ou l'étiologie d'un monde qui change. Unicité sémantique et pluralité référentielle, Mots. Les langages du politique, $\mathrm{n}^{\circ} 87$, juillet, Chrononymes. La politisation du temps, p. 57-69.

FrIzzı Géraldine, 2005, "La défense du nom des collectivités territoriales et le droit à la propriété intellectuelle», Revue générale des collectivités territoriales, $\mathrm{n}^{\circ} 32$ (1-2), p. 3-27.

FURET François, 1992, "L’Ancien Régime et la Révolution», P. Nora éd., Les lieux de mémoire, t. 3, Les France, Paris, Gallimard, p. 107-139.

FURET François, Ozouf Mona, 1992 [1988], Dictionnaire critique de la Révolution française (4 tomes), Paris, Flammarion.

GARDYPhilippe, 1987, «Réflexions à propos d'un toponyme : Larzac. Le nom impropre?», Cahiers de praxématique, $\mathrm{n}^{\circ} 8, \mathrm{p} .67-76$.

GARY-PRIEUR Marie-Noëlle, 1996, "Figurations de l'individu à travers différentes constructions du nom propre en français», Cahiers de praxématique, $\mathrm{n}^{\circ} 27$, p. 57-72.

Gauthier Gilles, 1992, "Nommer ou ne pas nommer. Un fondement rationnel de la pudeur journalistique », Communication, $\mathrm{n}^{\circ} 13$ (1), p. 15-44.

Gefrroy Annie, 1997, «Terreur et terrorisme. Les mots en héritage, du néologisme au concept», A. Gérard éd., La Vendée. Après la terreur, la reconstruction, Paris, Perrin, p.144-161.

Glatigny Michel, 2003, "L'article Arabe dans un certain nombre de dictionnaires français du Nicot au Grand Robert », Cahiers de lexicologie, n 83, p. 105-130.

GoNAC'H Jeanne, 2007, «Pratiques de redénomination des rues à Vitrolles», G. Cislaru et al. éd., L'acte de nommer. Une dynamique entre langues et discours, Paris, Presses de la Sorbonne nouvelle, p. 87-100.

GUÉRIN Olivia, 2006, «Un Français à la cour du Morho Naba», Mots. Les langages du politique, $n^{\circ} 82$, novembre, L'emprunt et sa glose, p. 23-35.

GUERRIN Christian, 1999, "Les processus redénominatifs dans les noms de communes françaises depuis 1943. Étude socio-toponymique de la variation dans la nomenclature administrative», S. Akin éd., Noms et re-noms. La dénomination des personnes, des langues et des territoires, Rouen, Publications de l'Université de Rouen, p. 209-227.

Guilhaumou Jacques, 2000, «Un nom propre en politique: Sieyès », Mots. Les langages du politique, $\mathrm{n}^{\circ} 63$, juillet, Noms propres, p. 74-86.

GuILLoN Jean-Marie, 2001, "Batailles de mémoires en Provence», J.-C. Bouvier, J.-M. Guillon éd., La toponymie urbaine. Significations et enjeux, Paris, L'Harmattan, p. 123-138.

HaRtog François, 2003, Régimes d'historicité, présentisme et expériences du temps, Paris, Le Seuil.

HENRY Marianne, 1985, «La production praxémique d'un toponyme: Ladrecht », Cahiers de praxématique, $\mathrm{n}^{\circ}$ 4, p. 41-64. 
HoNORÉ Jean-Paul, 2000, «Entre usage et héritage. Aspects formels du changement de nom », Mots. Les langages du politique, $\mathrm{n}^{\circ} 63$, juillet, Noms propres, p. 19-40.

Honoré Jean-Paul, Paveau Marie-Anne, PérIÈs Gabriel éd., 2000, Mots. Les langages du politique, $n^{\circ} 63$, juillet, Noms propres.

JouAnNa Arlette, 2002, "La notion de Renaissance. Réflexions sur un paradoxe historiographique», Revue d'histoire moderne et contemporaine, $\mathrm{n}^{\circ} 49$ (4) bis, p. 5-16.

JURET Étienne Abel, 1947, «La francisation des noms de personnes», Population, vol. 2, $n^{\circ} 3$, p. 451-464.

Kahlouche Rabah, 1999, "La dénomination-redénomination. Un lieu de conflit identitaire», S. Akin éd., Noms et re-noms. La dénomination des personnes, des langues et des territoires, Rouen, Publications de l'Université de Rouen, p. 183-188.

LAVELle Pierre, 2008, «Chrononymes japonais», Mots. Les langages du politique, n 87 , juillet, Chrononymes. La politisation du temps, p. 71-84.

LE BART Christian, 2000, «Nommer les hommes politiques. Identités prescrites, stratégiques, polémiques», Mots. Les langages du politique, $n^{\circ} 63$, juillet, Noms propres, p. 127-133.

LECOLLE Michelle, 2006, «Polyvalence des toponymes et interprétation en contexte», Pratiques, $\mathrm{n}^{\circ}$ 129-130, p. 107-122.

- 2004, "Toponymes en jeu. Diversité et mixage des emplois métonymiques de toponymes ", Studii si cercercerati filologica, ${ }^{\circ}$ 3, p. 5-13.

- 2001, "Personnification et métonymie dans la presse écrite. Comment les différencier? », Semen, $n^{\circ} 15$ (2), p. 97-112.

LefebVRe-Teillard Anne, 2000, "Le nom propre et la loi», Mots. Les langages du politique, $\mathrm{n}^{\circ} 63$, juillet, Noms propres, p. 9-18.

LEIMDORFER François, 2005, «Des villes, des mots, des discours», Langage et société, $\mathrm{n}^{\circ} 114$, p. 129-146.

- 2001, "Saint-Quentin en Yvelines" ou "Ville nouvelle" ? Le choix de nom comme choix de ville», Langage et société, $n^{\circ}$ 96, p. 71-103.

Leimdorfer François, Couret Dominique, KouAdio N'Guessan Jérémie, Soumahoro Christelle, 2002, «Nommer les quartiers d’Abidjan», C. Topalov éd., Les divisions de la ville, Paris MSH, p. 313-346.

Leroux Fabien, 2001, "Pour une onomastique des partis politiques. Éléments d'une étude des dénominations partisanes», mémoire de l’IEP de Paris, dir. Michel Offerlé.

Leroy Sarah, Siblot Paul, 2000, "L'antonomase entre nom propre et catégorisation sociale», Mots. Les langages du politique, $n^{\circ}$ 63, juillet, Noms propres, p. 89-104.

LEROY Sarah, 2004a, Le nom propre en français, Paris, Ophrys, $138 \mathrm{p}$.

- 2004b, De l'identification à la catégorisation. L'antonomase du nom propre en français, Louvain, Paris, Peeters.

LetTIerı Carmela, 2008, "L'Italie et ses Années de plomb. Usages sociaux et significations politiques d'une dénomination temporelle», Mots. Les langages du politique, $\mathrm{n}^{\circ} 87$, juillet, Chrononymes. La politisation du temps, p. 43-50. 
MARCot François éd., Dictionnaire historique de la Résistance, Paris, Laffont, notamment «Anthropologie de la vie résistante», p. 917-993.

MARTELClaude, 2001, "Auxgrands hommes, la cité reconnaissante? La patrotoponymie dans les Alpes-de-Haute-Provence», J.-C. Bouvier, J.-M. Guillon éd., 2001, La toponymie urbaine. Significations et enjeux, Paris, L'Harmattan, p. 75-87.

MAus Didier, FAVOreu Louis, PARODI Jean-Luc éd., 1992, L'écriture de la Constitution de 1958, Aix-en-Provence et Paris, Presses universitaires d'Aix-Marseille et Economica.

MEYER Jean, 1970, "Les ouvriers et la révolution mexicaine. Les bataillons rouges», Annales. Histoire, sciences sociales, $\mathrm{n}^{\circ} 25$ (1), p. 30-55.

Michonneau Stéphane, 2001, "Nouvelle toponymie, nouvelle identité. Le cas de la Barcelone des agrégations, 1897-1910», J.-C. Bouvier, J.-M. Guillon éd., 2001, La toponymie urbaine. Significations et enjeux, Paris, L'Harmattan, p. 151-169.

MıLo Daniel, 1986, «Le nom des rues», P. Nora éd., Les lieux de mémoire, t. 2, La Nation, Paris, Gallimard, p. 283-315.

Montécot Christiane, Osıpov Vladimir, VASSILAKı Sophie éd., Cahiers balkaniques, $\mathrm{n}^{\circ}$ 32, Autour du nom propre.

MULLER Pierre, 1999, "D'Instruction publique à Éducation nationale», Mots. Les langages du politique, $\mathrm{n}^{\circ}$ 61, décembre, L’École en débats, p. 85-104.

Nora Pierre éd., 1984-1992, Les lieux de mémoire (3 tomes, 7 volumes), Paris, Gallimard.

OfFERLÉ Michel, 1993, "Usages et usure de l'hérédité en politique », Revue française de science politique, $\mathrm{n}^{\circ} 43$ (5), p. 850-856.

PANICACCI Louis, 2001, "Les lieux de mémoire toponymiques de la Deuxième Guerre mondiale dans les villes azuréennes», J.-C. Bouvier, J.-M. Guillon éd., La toponymie urbaine. Significations et enjeux, Paris, L'Harmattan, p. 89-102.

PASQUINI Pierre, 2001, "Sorgues. Les mémoires croisées d'une cité vauclusienne», J.-C. Bouvier, J.-M. Guillon éd., La toponymie urbaine. Significations et enjeux, Paris, L'Harmattan, p. 111-122.

Pauleau Christine, 1997, "Calédonien et Caldoche», Mots. Les langages du politique, $n^{\circ}$ 53, décembre, La Nouvelle-Calédonie après les accords de Matignon, p. 48-65.

PAVEaU Marie-Anne, 2008, "Le toponyme, désignateur souple et organisateur mémoriel. L'exemple du nom de bataille », Mots. Les langages du politique, ${ }^{\circ} 86$, février, Toponymes. Instruments et enjeux, p. 23-35.

PÉRIÈs Gabriel, 1992, "L'Arabe, le Musulman, l'Ennemi dans le discours militaire de la "guerre révolutionnaire" pendant la guerre d’Algérie», Mots. Les langages du politique, $\mathrm{n}^{\circ}$ 30, mars, Images arabes en langue française, p. 53-70.

- 2000, "Au nom de l'ennemi. Tactiques de la parole doctrinale dans le discours militaire français», Mots. Les langages du politique, $n^{\circ}$ 63, juillet, Noms propres, p. 60-73.

Pernot Hélène, 2002, "L'appropriation du mot sud par les militants Sud-PTT», Mots. Les langages du politique, $n^{\circ} 68$, mars, Les métaphores spatiales en politique, p. 109-121.

- 2007, «Sud-PTT. La rencontre d'un mot et d'un projet syndical», P. Bacot, S. RémiGiraud éd., Mots de l'espace et conflictualité sociale, Paris, L'Harmattan, p. 257-265. 
Perrakı Vivi, 1990, "Du non-dit au cliché. Les avatars de Grande guerre et de Guerre mondiale», Mots. Les langages du politique, $\mathrm{n}^{\circ} 24$, septembre, Paroles de la Grande Guerre, p. 5-19.

PÉrus Jean, 1987, "Un emprunt au russe dans la terminologie politique des années trente : front populaire», Mots. Les langages du politique, $\mathrm{n}^{\circ} 15$, octobre, Comment nommer?, p. 189-192.

Pineira-Tresmontant Carmen, 2004, "Sémantique et Histoire. España et Españas dans le discours de Juan Carlos», Mots. Les langages du politique, $\mathrm{n}^{\circ} 75$, juillet, Émotion dans les médias, p. 61-73.

PIRES Mat, 2007, "Le détournement de sigle. Le cas de CPE», Langage et société, n 121-122, septembre-décembre, p. 289-303.

POCHARD Jean-Charles, 2007, "Points cardinaux et métonymies géopolitiques. Est/ Ouest, Nord/Sud», P. Bacot, S. Rémi-Giraud éd., Mots de l'espace et conflictualité sociale, Paris, L'Harmattan, p. 267-280.

RANGEL Vicente Montserrat, 2004, «Nom propre et dialogisme. La construction de la représentation de Napoléon Bonaparte en Espagne et en France», A. Cassanas, A. Demange, B. Laurent, A. Lecler éd., Dialogisme et nomination, Montpellier, Presses universitaires de Montpellier 3, p. 199-214.

Regault Jean-Marc, 1996, «Tahiti, EFO, Polynésie française, Ao Maohi.... Quel nom pour ce territoire d'outre-mer? Un essai d'onomastique politique », Revue juridique et politique. Indépendance et Coopération, $\mathrm{n}^{\circ}$ 3, p. 296-309.

Rey-Debove Josette, Rey Alain, Bellefonds Christine (de), 2007, Le Robert encyclopédique des noms propres, Paris, Le Robert.

RichaRD Éliane, 2001, "Femmes dans la rue ou les "trous de mémoire" de la ville. L'exemple marseillais », J.-C. Bouvier, J.-M. Guillon éd., 2001, La toponymie urbaine. Significations et enjeux, Paris, L'Harmattan, p. 103-110.

Rostaing Charles, 1965, Les noms de lieux, Paris, PUF.

Ruiz Juan Pro, 2006, «Figure du cacique, figure du caudillo. Les langages de la construction nationale en Espagne et en Argentine, 1808-1930 ", Genèses, n 62, mars, p. 27-48.

SARFATI Georges Elia, 1997, "L'étymologie sociale du mot Juif», Mots. Les langages du politique, $\mathrm{n}^{\circ}$ 50, mars, Israël-Palestine. Mots d'accord et de désaccord, p. 138-142.

- 2000, "Le statut lexicographique du nom propre. Remarques méthodologiques et linguistiques», Mots. Les langages du politique, $n^{\circ} 63$, juillet, Noms propres, p. 105-124.

SAUNIER Pierre-Yves, 1990, "Tempêtes dans une petite plaque d'émail bleu. Les noms de rue à Lyon entre 1814 et 1914 ", Cahiers d'histoire, n 4 .

- 1993, "La ville et ses découpages», Mélanges de l'École française de Rome. Italie et Méditerranée, $\mathrm{n}^{\circ} 105$ (2), p. 375-403.

Sıв Lot Paul éd., 1987, Cahiers de praxématique, $n^{\circ} 8$, Théories et fonctionnements $d u$ nom propre.

SIBLOT Paul, 1987, «De la signifiance du nom propre», Cahiers de praxématique, n 8, p. 197-114.

- 1989, «Noms propres et mains sales. De l'inscription des luttes sociales dans les praxèmes en nomination individuelle », Langages, n² 24-93, p. 64-83. 
- 1998, "Algérien dans l'imbroglio des dénominations», Mots. Les langages du politique, $\mathrm{n}^{\circ}$ 57, décembre, Algérie en crise. Entre violence et identité, p. 7-27.

- 2004, "Du dialogisme de la nomination», A. Cassanas, A. Demange, B. Laurent, A. Lecler éd., Dialogisme et nomination, Montpellier, Presses universitaires de Montpellier 3, p. 331-337.

- 2007, «Entre Orient et Occident. L'espace du sens», P. Bacot, S. Rémi-Giraud éd., Mots de l'espace et conflictualité sociale, Paris, L'Harmattan, p. 205-213.

Siblot Paul, LeRoy Sarah, 2000, "L'antonomase entre nom propre et catégorisation nominale», Mots. Les langages du politique, $\mathrm{n}^{\circ} 63$, juillet, Noms propres, p. 89-104.

SOMMERER Erwan, 2008, "Une démocratie illégitime. La década infame dans le discours populiste argentin », Mots. Les langages du politique, $n^{\circ} 87$, juillet, Chrononymes. La politisation du temps, p. 27-41.

SUBLET Jacqueline, 1993, Le voile du nom. Essai sur le nom propre arabe, Paris, PUF, 1991. TISSIER Yves, 2004 [2 édition], Dictionnaire de l'Europe. États d'hier et d'aujourd'hui de 1789 à nos jours, Paris, Vuibert.

Topalov Christian, 2002, "Les divisions de la ville. Une approche par les mots", C. Topalov éd., Les divisions de la ville, Paris, MSH, p. 1-5.

TOURNIER Maurice, 1989, "Le Grand Soir, un mythe de fin de siècle », Mots. Les langages du politique, $\mathrm{n}^{\circ} 19$, juin, Bataille de mots autour de 1900, p. 79-94.

- 2000, «Europe, terme et terre d'immigration», Mots. Les langages du politique, $n^{\circ} 63$, juillet, Noms propres, p. 141-146.

- 2000, "Franc, stéréotype du Français», Mots. Les langages du politique, n 64 , décembre, Autour d'une crise franco-australienne. Stéréotypies xénophiles et xénophobes, p. 136-141.

- 2005, "De Barbare à Babel, des sons qui bredouillent et excluent», Mots. Les langages du politique, $\mathrm{n}^{\circ} 78$, juillet, Usages politiques du genre, p. 131-142.

- 2005, "La graisse vorace. Petits mythes populaires au service des désignations sociopolitiques à la fin du $19^{\mathrm{e}}$ siècle», Langage et société, $\mathrm{n}^{\circ} 113$, septembre, p. $94-123$.

VALLET Odon, 1989, «Le mot Parlement», Mots. Les langages du politique, $n^{\circ} 19$, juin, Bataille de mots autour de 1900, p. 97-98.

- 1998, "Les noms de l'innommable», Mots. Les langages du politique, ${ }^{\circ}{ }^{56}$, septembre, La Shoah : silence... et voix, p. 138-141.

VAN De VeLDE Danièle, 2000, «Existe-t-il des noms propres de temps?», Lexique, nº 15, p. $35-45$.

VeNIARD Marie, 2003, "Guerre dans le discours de presse sur les conflits armés. Orientation argumentative et marque de point de vue», Communication aux Rencontres de l'école doctorale Langage et Langues (ED 268), mai 2003, Université Paris 3 Sorbonne nouvelle, disponible sur le site des RJC, www.cavi.univparis3.fr/ ilpga/ED/activites/RJC2003_actes/index.html

- 2004, "Les désignations du conflit du Golfe dans la presse. Un miroir du conflit sur le terrain?», A. Cassanas, A. Demange, B. Laurent, A. Lecler éd., Dialogisme et nomination, Montpellier, Presses universitaires de Montpellier 3, p. 99-111. 
VERNIER Bernard, 1989, "Fétichisme du nom, échanges affectifs intra-familiaux et affinités électives », Actes de la recherche en sciences sociales, $n^{\circ} 78$, p. 2-17.

VIDAL Fabrice, 2004, "Construction des identités et légitimation à travers les titres des listes candidates aux élections municipales à Lyon sous la Ve République», mémoire de DEA de Science politique, Institut d'études politiques de Lyon, dir. Paul Bacot.

WAUTHION Michel, 1998, «Noms de pays. Le nom changeant de ses institutions », Revue nouvelle, $\mathrm{n}^{\circ} 107$ (2), p. 49-65.

Weber Eugen, 1986, «L’Hexagone», P. Nora, Les lieux de mémoire, t. 2, La Nation, Paris, Gallimard, p. 97-116.

WEIS Béatrice, 1993, "Essai sur la francisation des toponymes en Alsace depuis la royauté jusqu'à nos jours », Nouvelle revue d'onomastique, $n^{\circ}$ 21-22, p. 109-112.

Weitman Sasha, 1987, "Prénoms et orientations nationales en Israël, 1882-1980», Annales. Histoire, Sciences sociales, $\mathrm{n}^{\circ}$ 42, p. 879-900.

YASRI-LABRIQUE Éléonore, 2008, "La Turquie, terre eurasiatique et république bicéphale. Ankara et Istanbul à travers la presse», Mots. Les langages du politique, $n^{\circ} 86$, février, Toponymes. Instruments et enjeux, p. 37-52. 